\title{
Quelques solutions aux problèmes posés par les grandes vannes d'aménagements hydrauliques
}

\author{
Some solutions to the problems of major sluices \\ in hydraulic installations
}

\author{
A. Josserand et J. Delaroche \\ Ingénieur Vannes et Conduites Forcées \\ Neyrpic Grenoble
}

Les aménagements hydrauliques modernes comprennent des vannes de fond et de surface de dimensions de plus en plus importantes et soumises à des efforts considérables. Il en résulte, pour le Constructeur, l'obligation de résoudre les problèmes posés par la réalisation et le bon fonctionnement de ces vannes.

Les problèmes hydrauliques peuvent être analysés, des calculs peuvent être faits, mais il est difficile, sinon impossible, de prévoir toutes les conditions d'écoulement auxquelles les vannes peuvent etre soumises. En dépit des précautions prises, des problèmes imprévus peuvent se présenter, rendant impossible la manœuvre des vannes et pouvant aller jusqu'à mettre en danger les ouvrages.

L'analyse des solutions déjà adoptées permet de résoudre une grande partie des problèmes mais on doit fréquemment compléter cette étude par des essais sur modèle réduit.

Le modèle réduit, bien conçu, est un outil précieux pour le projeteur. On peut déceler les zones où des incidents pourraient se produire, étudier les améliorations à apporter aux tracés des ouvrages pour éviter ou réduire des phénoménes dangereux.

Il permet également la mesure des efforts hydrauliques sollicitant les organes mobiles. Par l'enregistrement de ces efforts en cours de manœuvre, on peut analyser les phénomènes très localisés (instabilités d'écoulement, d'aération, pulsations de pression etc...).

En dehors des problèmes purement hydrauliques, il reste également à résoudre les problèmes de réalisation.

La fabrication des grandes vannes pose les problèmes classiques des grandes constructions mécano-soudées, nous examinerons plus particulièrement ceux plus spécifiques aux vannes et parmi eux:

- l'alignement des articulations d'un clapet de grande largeur ;

- l'assemblage entre éléments des structures;

- certains dispositifs d'étanchéité pour grandes vannes.
Alignement des articulations des clapets de grande largeur

Parmi les divers types de vanne, le clapet permet un réglage fin du niveau d'une retenue, du débit déversé et facilite l'évacuation des corps flottants. Si sa hauteur n'est jamais très grande, sa largeur peut être très importante. Il est souvent utilisé pour accroître la capacité de stockage des réservoirs anciens, sans modifier les niveaux extrêmes de crues. Son utilisation permet des installations esthétiques.

Cette dernière décennie Neyrpic et BVS ont réalisé un grand nombre de clapets dont certains de grande largeur. - Couzon : 3 clapets $35 \mathrm{~m} \times 4,8 \mathrm{~m}$. Sur la Saône. - Charnay : 3 clapets $35 \mathrm{~m} \times 3,1 \mathrm{~m}$. Sur la Saône. - Pagny: 3 clapets $36,5 \mathrm{~m} \times 3,0 \mathrm{~m}$. Sur la Saône. - Ruacana : 5 clapets $56 \mathrm{~m} \times 3,5 \mathrm{~m}$. Cunene. (Afrique du Sud).

- Idaho Falls : entre autres 2 clapets $45 \mathrm{~m} \times 3 \mathrm{~m}$. Sur la Snake River (U.S.A.).

Un clapet consiste en un corps de torsion (plan du barrage de Pagny Fig. 1), s'étendant sur toute la largeur obturée et dont le bordé amont, de forme appropriée sert de coursier déversant. Un certain nombre de voiles transversaux assurent l'indéformabilité de la section et transmettent les poussées hydrauliques au béton en s'appuyant sur des paliers à coussinets généralement en bronze (Dessin d'un palier Fig. 2).

Le nombre de paliers est souvent assez élevé :

- Couzon 13

- Ruacana 18

- Idaho 18

La raideur des ossatures implique un alignement précis des paliers en particulier entre paliers voisins, pour éviter des efforts secondaires importants dans les paliers, des suppléments de contraintes dans les ossatures et d'efforts dans les mécanismes de mancuvre. 
Un écart d'alignement de $0,5 \mathrm{~mm}$ est le maximum admissible.

Chaque palier est indépendant et possède les dispositifs de réglage qui permettent son alignement, la difficulté est au niveau de l'alignement des alésages dans les voiles transversaux du volet compte tenu de la construction en éléments soudés sur chantier (dessin d'un palier).

Pour éviter tout problème, l'alésage final des voiles transversaux est réalisé au chantier au moyen d'une unité d'alésage portative dont la broche est soutenue et centrée dans les paliers, ceux-ci étant réglés avec soin préalablement. (Schéma de montage Fig. 3).

Ce procédé a déjà été utilisé pour Pagny, Ruacana et le sera au printemps prochain à Idaho Falls.

\section{Assemblage entre éléments de structures des vannes-wagon}

Pour tenir compte des défauts de construction et de montage des rails de roulement et des structures, Neyrpic réalise les vannes-wagon en éléments à 4 galets (ou 4 boggies). L'ossature de ces éléments est du type à profil ouvert donc souple en torsion. Avec cette conception, la répartition réelle des charges entre galets est très voisine de la répartition théorique et n'est que faiblement affectée par la sortie de la génératrice de roulement d'un galet hors du plan défini par celles des trois autres.

Les éléments de vanne sont assemblés entre eux par des dispositifs assurant la transmission des efforts dans le plan de la vanne tout en permettant la rotation et les déplacements relatifs (voir Fig. 4 et 5) sans introduction d'efforts secondaires importants.

(A noter le dispositif de réglage avec araldite pour assurer un plan d'appui à partir de surfaces brutes de mécano-soudage).

Outre son intérêt du point de vue mécanique des constructions, cette conception permet d'expédier des éléments entièrement finis d'atelier. La liaison au chantier se limite à la mise en place de quelques pièces. Il n'y a pas de précautions spéciales à prendre pour la soudure (température minimum - préchauffage pour aciers spéciaux, contrôles radiographiques).

La liaison s'effectuant en dehors des rainures, l'assemblage des éléments peut être réalisé sur le seuil en utilisant des engins de montage de puissance réduite par rapport à celle de l'appareil de manœuvre de service (vérin par exemple).

\section{Dispositifs d'étanchéité pour grandes vannes}

M. Milan a consacré un chapitre de son exposé aux vibrations des dispositifs d'étanchéité des vannes. Il a particulièrement recommandé d'éviter les faibles ouvertures qui peuvent les provoquer et les entretenir. Ces faibles ouvertures peuvent ètre dues aux déformations des structures sous charge si les dispositifs d'étanchéité sont mal conçus.

Nous allons présenter quelques dispositions permettant une étanchéité permanente.

\section{1. - Etanchéité vanne segment de grande largeur et étan- chéité sur seuil}

Extrait du plan de Vaugris (Fig. 6)

Dimensions des vannes: largeur $21,0 \mathrm{~m}$, hauteur: $12,80 \mathrm{~m}$

Le couteau de la vanne a été conçu pour obtenir un appui continu sur le seuil sous la charge nominale.

Les croquis montrent clairement les déplacements des diverses sections. On peut constater que la garniture d'étanchéité est toujours en contact avec le seuil, quelle que soit la charge d'eau. Le joint en note de musique a été choisi pour sa souplesse et sa fixation a été prévue en conséquence.

On voit qu'avec cette disposition, on ne peut pas se permettre de faibles ouvertures prolongées. Les schémas des armoires et les automatismes sont prévus en conséquence.

\section{2. - Etanchéité frontale de vanne de fond}

\section{Extrait du plan de Granadilla (Fig. 7 et 8 )}

Les vannes aval de groupes peuvent fonctionner en organe de coupure et également en batardeau aval.

Leurs caractéristiques sont :

- longueur : $8,40 \mathrm{~m}$.

- hauteur : $5,319 \mathrm{~m}$.

- charge d'eau maxi/seuil : $38,00 \mathrm{~m}$.

En vanne de coupure la flèche est de $10 \mathrm{~mm}$ dans le sens amont-aval et en batardeau du même ordre (sens aval-amont).

Le déplacement entre les 2 positions extrêmes est de $22 \mathrm{~mm}$ compte tenu d'un jeu de $2 \mathrm{~mm}$ dans la rainure. Pour permettre à la garniture frontale de rester toujours comprimée, sur la face d'appui, celle-ci est mise en pression soit par l'amont soit par l'aval à l'aide d'un distributeur à clapets figure 9 .

\section{3. - Etanchéité au seuil de porte d'écluse de $24 \mathrm{~m}$}

\section{Plan d'IFFEZHEIM (Fig. 10)}

La flèche de l'ossature en charge est de $42 \mathrm{~mm}$. Les formes et les dimensions de la garniture elle-même ne permettent pas ce déplacement.

Il a été nécessaire de la placer sur un support mobile, ce qui implique une garniture additionnelle. La pression d'eau tend à appliquer la garniture d'étanchéité contre la pièce scellée. 


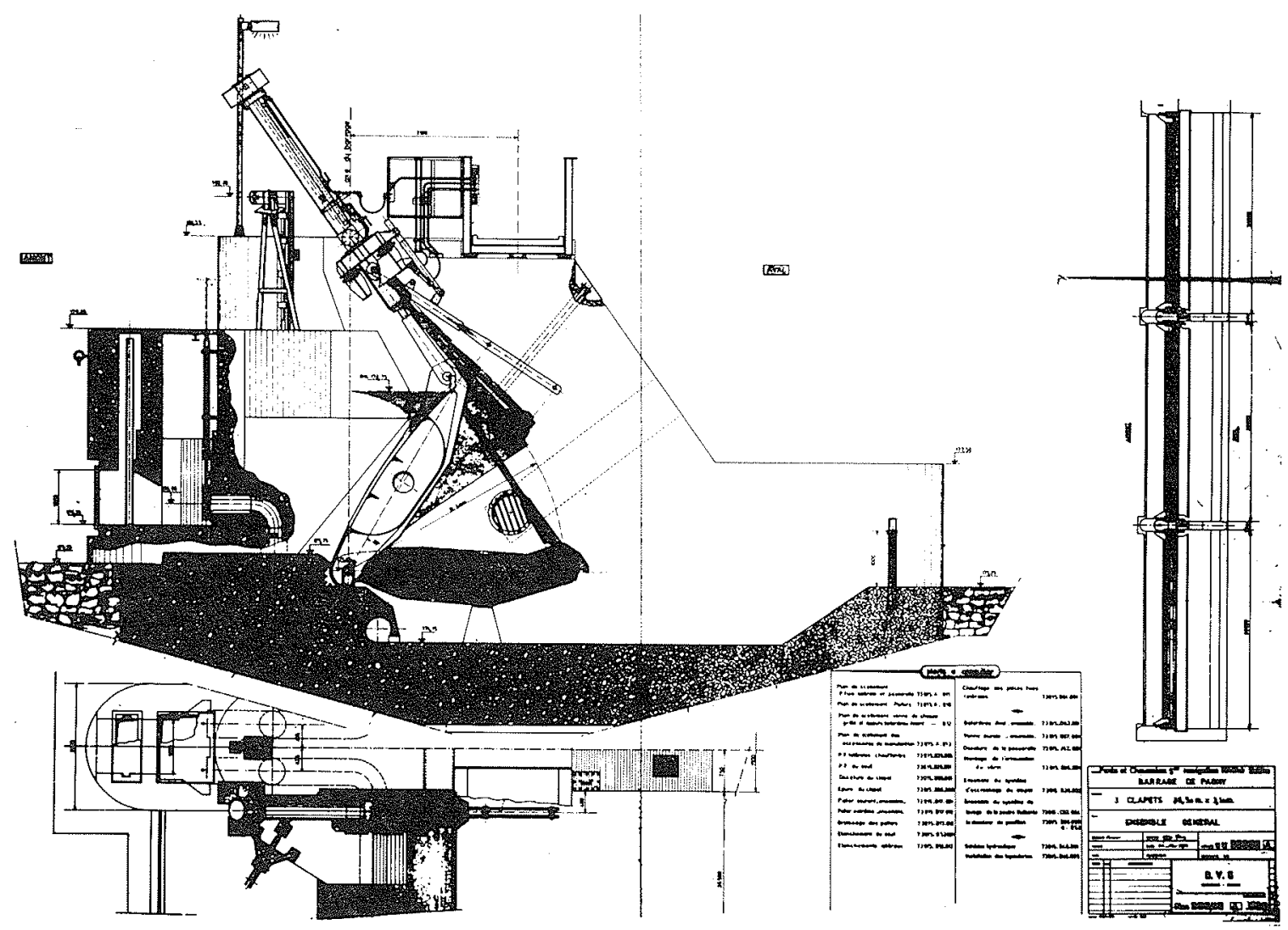

Figure 1

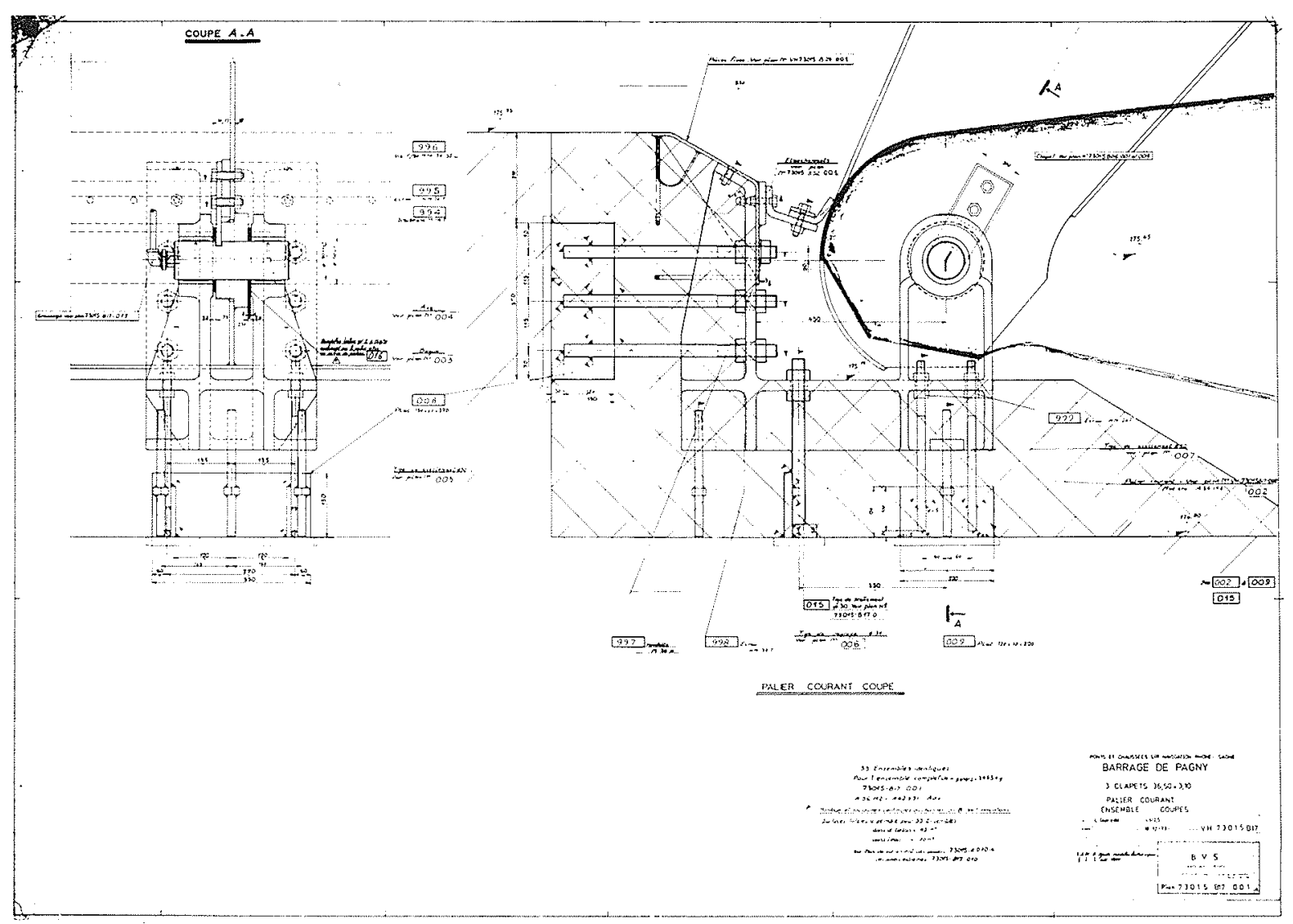

Figure 2 


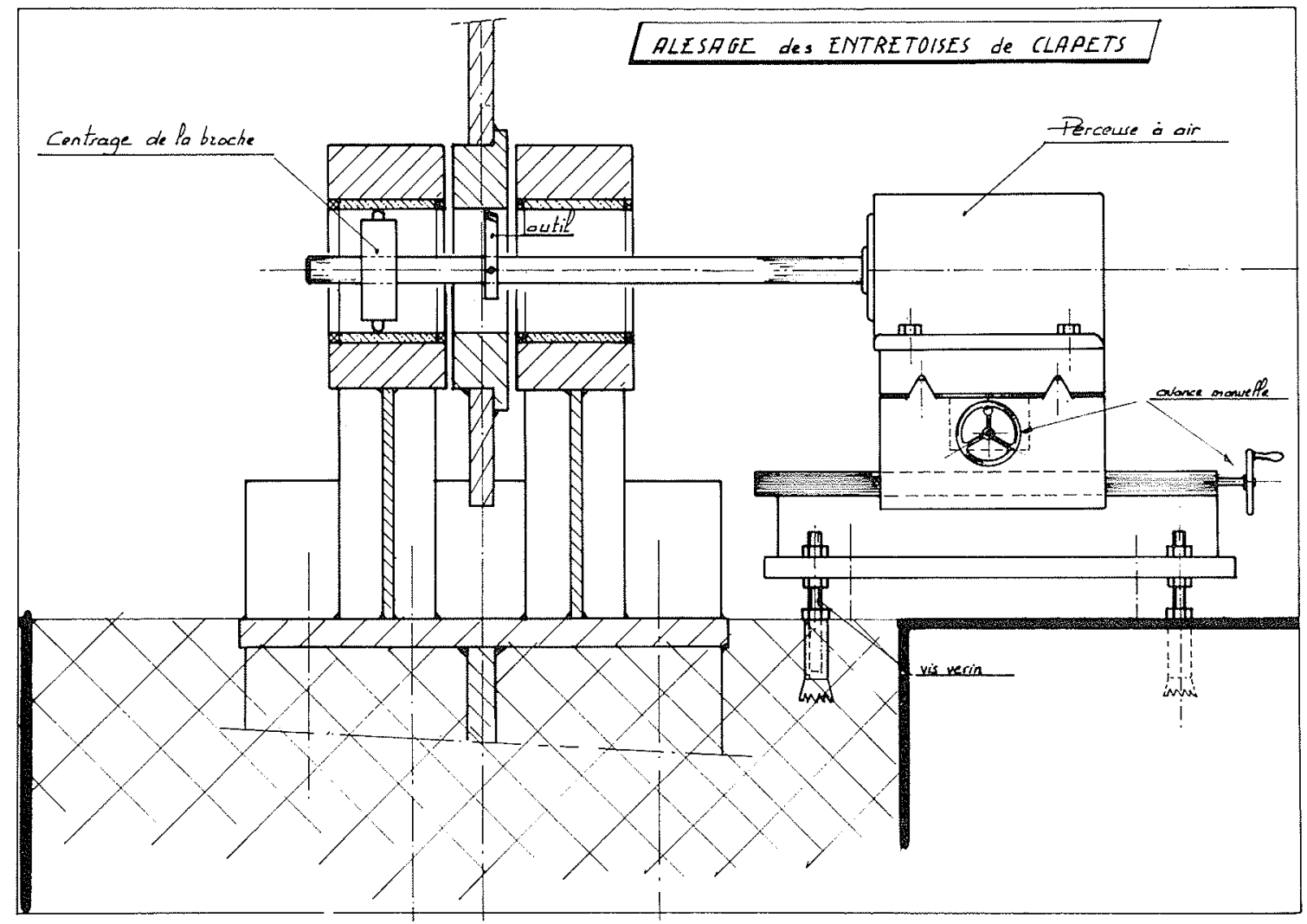

Figure 3
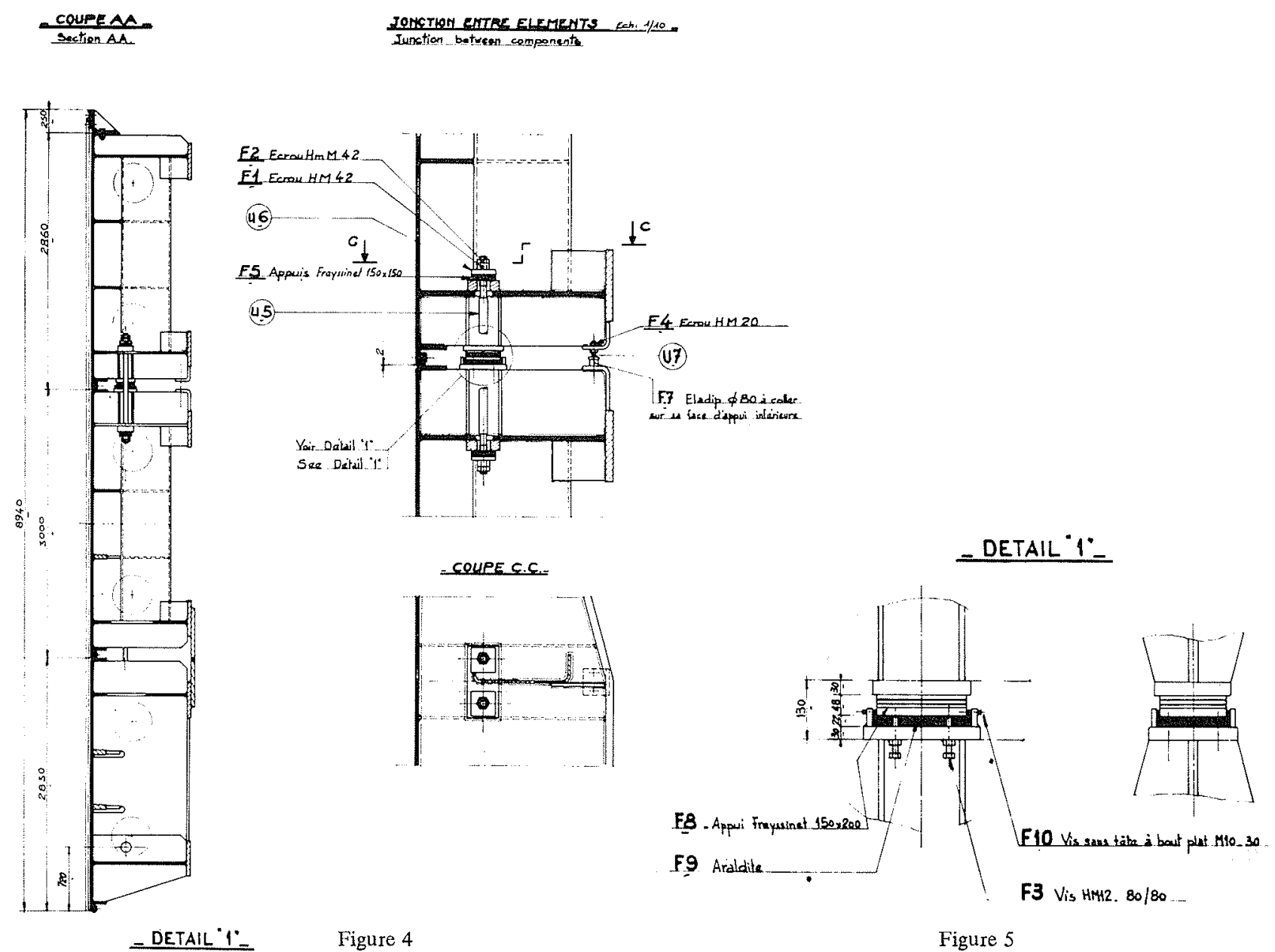

Figure 5 

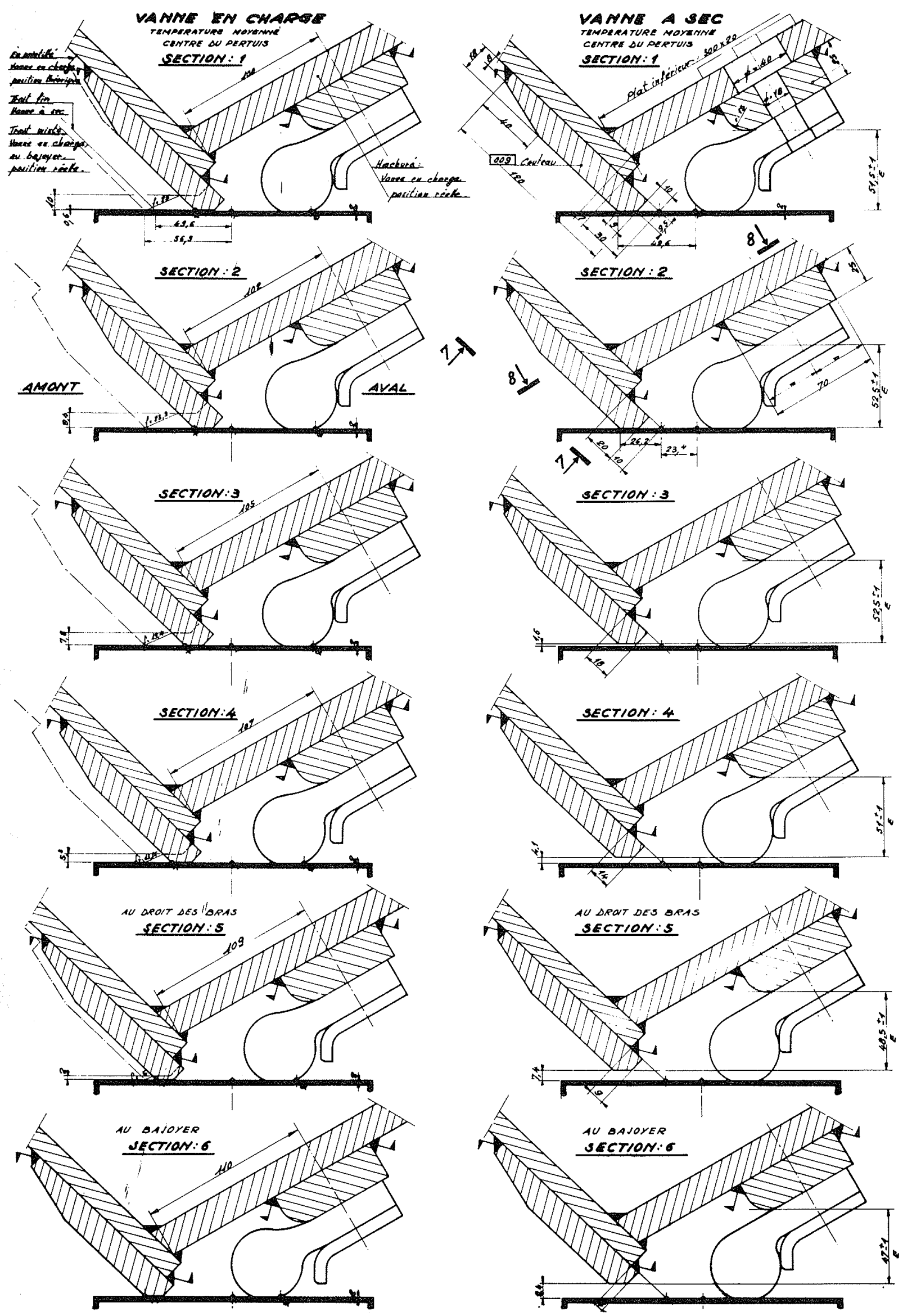

Figure 6 


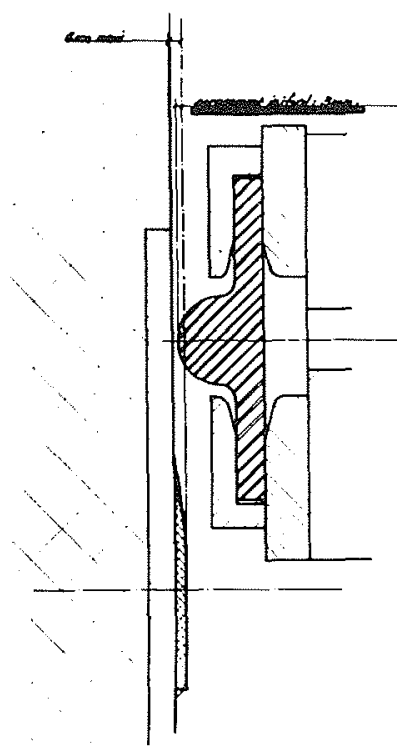

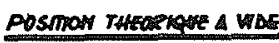

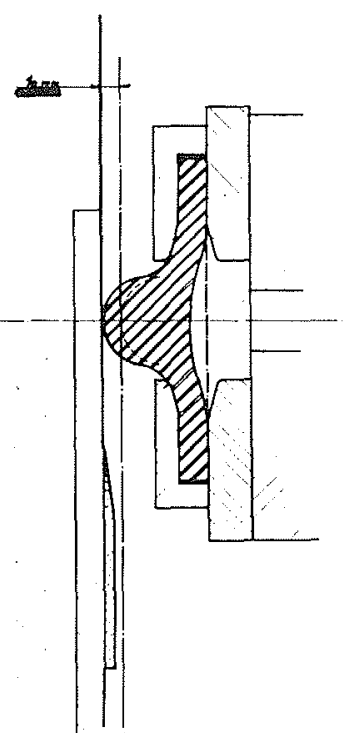

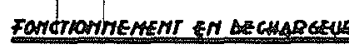

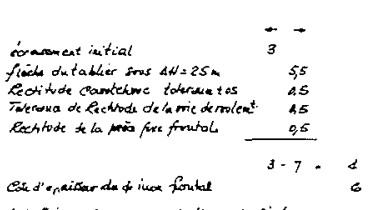

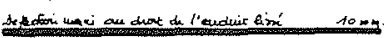

Figure 7

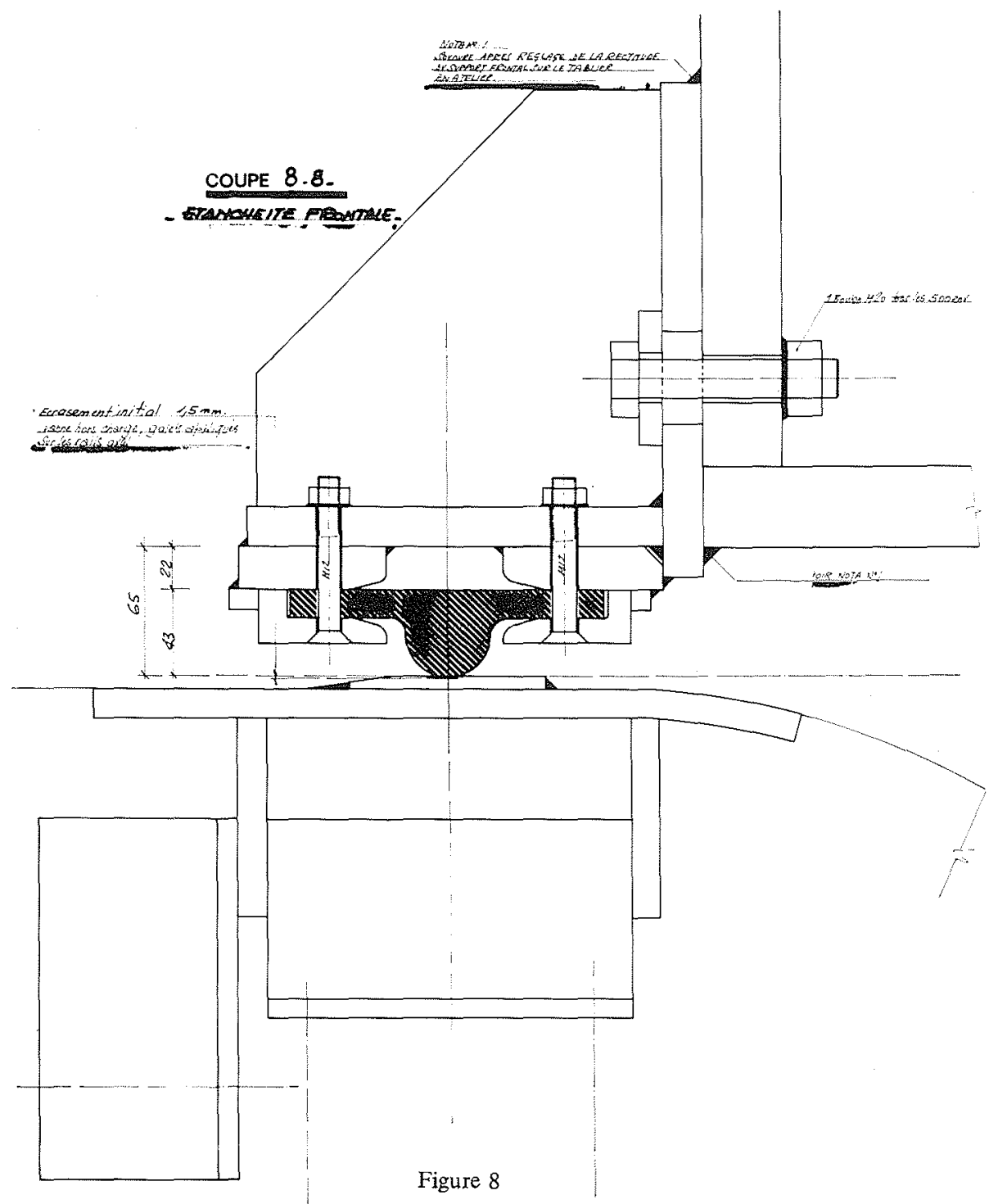



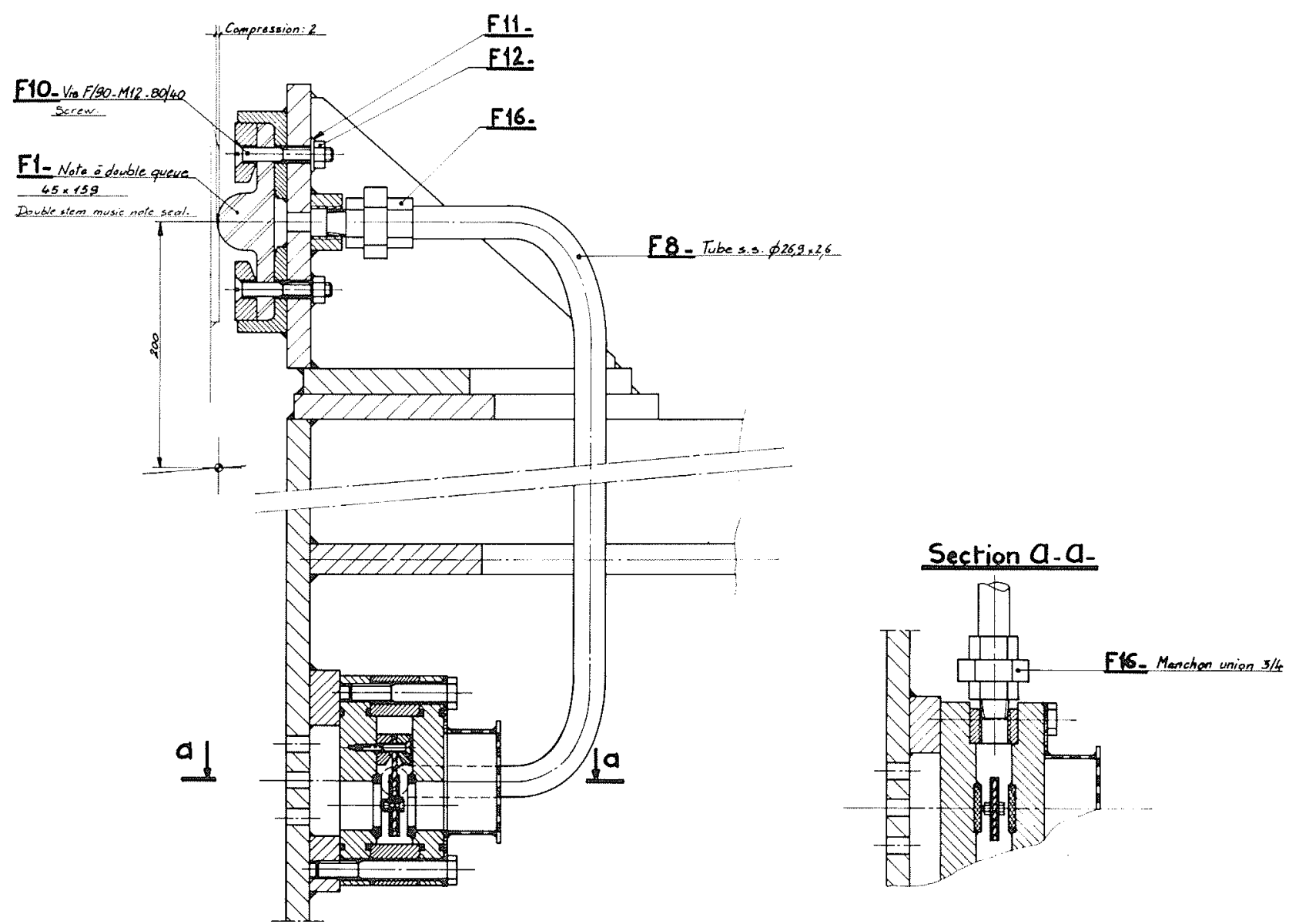

Figure 9

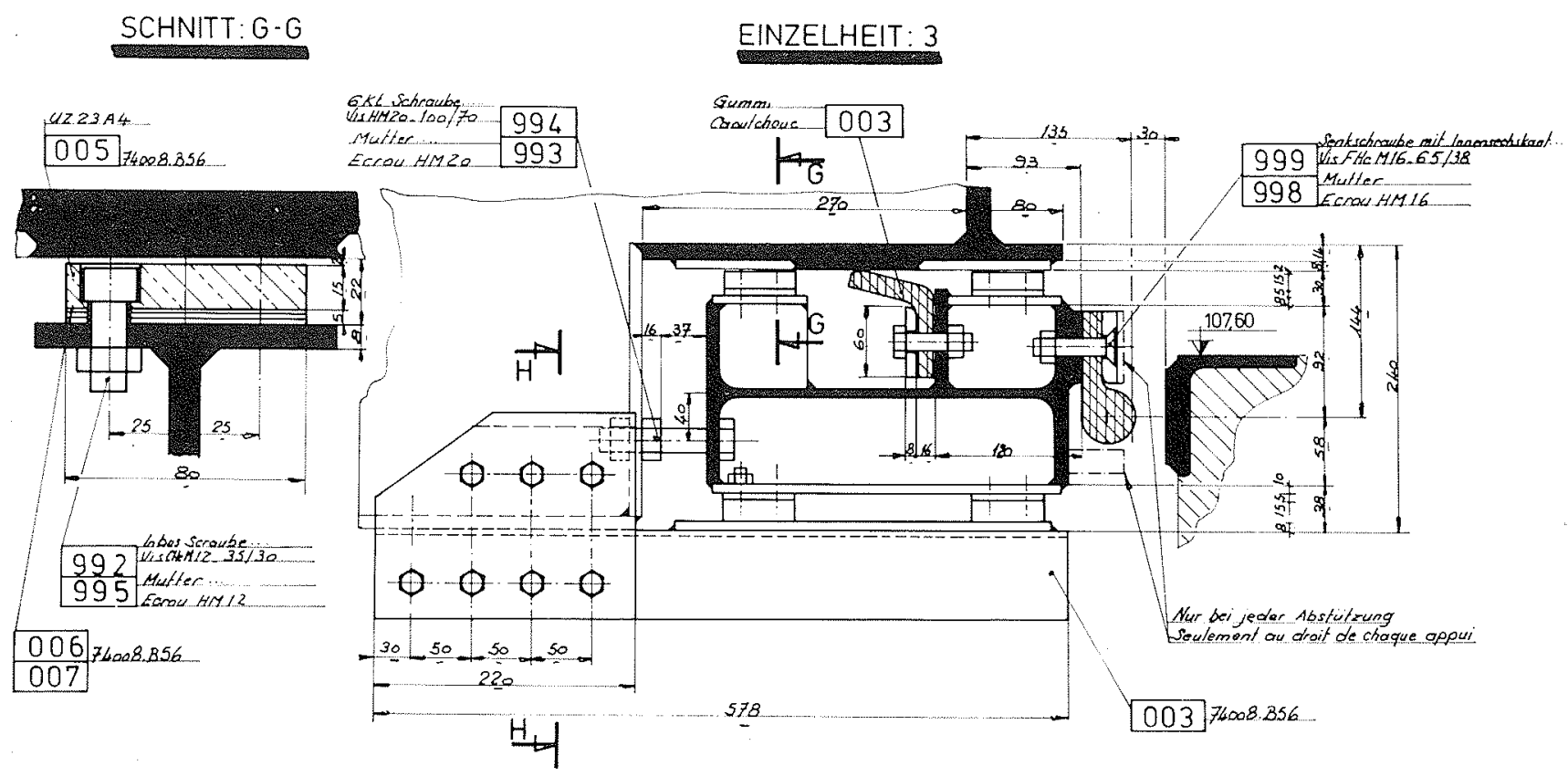

Figure 10 


\section{Discussion}

Président : M. COMBELLES

M. le Président. - Je vous remercie pour la clarté de votre exposé et pour votre exactitude.

Tout à l'heure vous nous avez dit qu'il fallait éviter de mettre les vannes-segments à faible ouverture. Qu'entendez-vous par "faible ouverture"?

M. DELAROCHE. - Des ouvertures de 10 à $15 \mathrm{~cm}$ pour des vannes de ce genre.

M. LACOSTE. - Dans les installations hydrauliques, la plupart des organes de coupure installés sur les adductions manœuvrent habituellement en milieu équilibré, la coupure de sécurité en charge n'étant que très exceptionnelle.

Toutefois sur les installations basse chute, certaines vannes, par exemple les vannes wagon d'alimentation des écluses et les vannes aval des groupes bulbes à pales et vannages fixes, manœuvrent normalement en charge.

Connaît-on l'influence de ce mode de fonctionnement sur les phénomènes de fatigue sous l'influence des vibrations et sur les usures des étanchéités et pièces fixes de roulement ?

M. DELAROCHE. - En ce qui concerne les vibrations, que les vannes fonctionnent peu ou souvent, il faut prendre les précautions nécessaires pour les empêcher de vibrer.

L'usure des étanchéités pose un problème notamment pour les étanchéités frontales des vannes aval; le type de garniture présenté devrait éviter le frottement continu donc diminuer l'usure.

Par contre, en ce qui concerne les usures de chemin de roulement, c'est autre chose : c'est la définition des charges admissibles sur les roues.

Les charges qui sont actuellement admises ne tiennent pas toujours compte de la fréquence des manœuvres. Là, il faudrait que les Cahiers des Charges soient modifiés de manière à ce que les constructeurs respectent d'autres conditions de charge sur les roues qui évitent l'usure.

M. CRAVERO. - Ma question s'adresse à M. ROCCHI. Elle concerne les travaux d'Hydroart sur les vannes sphériques destinées à couper les débits des pompes-turbines et dont l'organe de réglage est un anneau mobile.
A Electricité de France, nous avons constaté en exploitation que les premiers millimètres d'ouverture de l'anneau mobile de réglage sont d'une importance considérable quant au régime vibratoire.

Est-ce que les travaux d'adjonction de grille dissipatrice vous ont permis de montrer sur modèle qu'il y avait une diminution des régimes vibratoires et des sources d'excitation?

M. ROCCHI. - Nous n'avons pas encore terminé nos essais. Nous en sommes au stade du laboratoire. Nous avons des espoirs, mais nous n'avons pas encore de certitudes actuellement.

D'autre part, vous avez dit que les anneaux ont été source de certains problèmes à $\mathrm{EDF}$.

M. CRAVERO. - Ce ne sont pas des problèmes d'étanchéité ; ce sont des problèmes de vibrations de nature hydraulique.

M. ROCCHI. - Cela dépend d'un certain nombre de facteurs. Cela dépend de l'effort qu'on va appliquer aux deux chambres pour le mouvement. Les anneaux ne sont pas faits partout de la même façon. Cela dépend de la forme de l'anneau et de la forme des chambres.

Il faudrait comparer les pièces et étudier les phénomènes sur un anneau, puis sur un autre. Il faudrait préciser cela.

Nous pouvons dire que les essais sur les modèles et les essais sur les prototypes n'ont pas fait apparaître, jusqu'à présent, de véritables raisons pour qu'on s'occupe de ce phénomène. Il faudrait voir différentes applications.

Ce que vous aviez vu a été fait à Charpira. Il y avait un vent de $3 \mathrm{~m}$ avec $300 \mathrm{~m}$ de charge. On a fermé. Il a fallu faire plusieurs mancuvres, et l'on n'a pas constaté ce défaut.

M. le Président. - Après avoir félicité les auteurs et remercié les animateurs de ce débat il me semble, pour conclure, que les différentes interventions ont montré à tout le monde que l'hydraulique était encore difficile, et que tous les problèmes ne pouvaient pas être résolus à coup d'ordinateurs. L'expérience des constructeurs est un gage important du succès et du mode de correction des petits ennuis qui peuvent se produire à la mise en service des matériels à utiliser. 